Research Paper

\title{
Effectiveness of Eccentric Exercise and a Vibration or Cryotherapy Program in Enhancing Rectus Abdominis Muscle Thickness and Inter-Rectus Distance in Patients with Chronic Mid-Portion Achilles Tendinopathy: A Randomized Clinical Trial
}

\author{
Carlos Romero-Morales ${ }^{1}$, Pedro Javier Martín-Llantino1 ${ }^{1}$, César Calvo-Lobo ${ }^{2 凶}$, Hector Beltran-Alacreu ${ }^{3,4}$, \\ Daniel López-López ${ }^{5}$, Rubén Sánchez-Gómez ${ }^{1}$, David Rodríguez-Sanz ${ }^{1}$ \\ 1. Faculty of Sport, Universidad Europea, Villaviciosa de Odón, Madrid, Spain. \\ 2. Nursing and Physical Therapy Department, Faculty of Health Sciences, Institute of Biomedicine (IBIOMED), Universidad de León, Ponferrada, Spain. \\ 3. Departamento de Fisioterapia. Centro Superior de Estudios Universitarios La Salle. Universidad Autónoma de Madrid. Spain. \\ 4. Motion in Brains Research Group. Instituto de Neurociencias y Ciencias del Movimiento. Centro Superior de Estudios Universitarios La Salle. Universidad \\ Autónoma de Madrid. \\ 5. Department of Health Sciences; Research, Health and Podiatry Unit. Faculty of Nursing and Podiatry, Universidade da Coruña, Ferrol, Spain.
}

$\square$ Corresponding author: Cesar Calvo Lobo. PhD, MSc, PT. Nursing and Physical Therapy Department, Institute of Biomedicine (IBIOMED), Faculty of Health Sciences, University of León, Av. Astorga, s/n, 24401 Ponferrada, León, Spain. Email: cecalvo19@hotmail.com

(c) Ivyspring International Publisher. This is an open access article distributed under the terms of the Creative Commons Attribution (CC BY-NC) license (https://creativecommons.org/licenses/by-nc/4.0/). See http://ivyspring.com/terms for full terms and conditions.

Received: 2018.07.20; Accepted: 2018.10.18; Published: 2018.11.23

\begin{abstract}
Purpose: Abdominal muscles are key in maintaining body stability and balance and an improvement in the functioning of these muscles could influence the rehabilitation process in lower limb pathologies such as Achilles Tendinopathy (AT). The aim was to explore whether calf eccentric exercise (EE) with vibration training was more effective at causing adaptation to the rectus anterior (RA) thickness and inter-rectus distance (IRD) than calf EE with cryotherapy.

Methods: The investigation was a single-blinded, randomized, controlled clinical trial (NCT03515148). Sixty-one individuals diagnosed with mid-portion AT were recruited and divided in two groups: group A $(n=30)$ followed an EE with vibration program and group $B(n=31)$ an EE program with cryotherapy, for 12-weeks. RA muscle thickness and IRD were measured in maximal isometric contraction and at rest as an indication of superficial abdominal muscle activation.

Results: IRD measures showed a significant $(P<0.05)$ decrease at baseline, 4 and at 12 -weeks in both groups, but no significant differences were observed between the intervention groups. RA thickness was significantly increased $(P<0.05)$ in measures at baseline, 4 and 12 -weeks showed a significant increase in maximal isometric contraction and at rest in favor of the EE vibration program group.

Conclusions: The present study showed a RA thickness increase in both groups in favor of the EE vibration program with respect to cryotherapy added to EE in short and mid term in maximal isometric contraction and at rest in subjects with chronic mid-portion AT. IRD was decreased in both groups without between-groups differences.
\end{abstract}

Key words: eccentric exercise, rectus abdominis, tendinopathy

\section{Introduction}

Achilles tendinopathy (AT) is a very common disease in athletes as well as in the general population [1]. This condition is characterized by pain, thickening and dysfunction of the Achilles tendon, which is one of the largest and strongest tendons in the lower limb and is highly susceptible to tendinopathy [2]. The 
prevalence and incidence rates of AT were 2.35 and 2.16 per 1000 person-years, respectively [3]. This common overuse injury is often difficult to treat, leading to a negative impact on sports participation as well as overall health and quality of life, sports, quality of life and at work [4].

Several studies have shown that AT leads to an increased eversion range of motion (ROM) in the rear foot, a shortened maximum lower leg abduction, a decreased ankle joint dorsiflexion velocity and a decreased knee flexion [5]. In addition, negative differences in ground reaction forces, dynamic plantar pressure and tibial external rotation have also been reported in patients with AT [5]. Moreover, a high body mass index (BMI) and disorders in blood flow and a sedentary lifestyle are also considered risk factors [6]. The term "tendinopathy" has been adopted following the latest advances in this area replacing the term "tendinitis", due to the absence of prostaglandin-meditated inflammation $[7,8]$. Several studies suggest that oral non-steroidal antiinflammatory drugs (NSAIDs) could help reducing the symptoms in individuals who suffer some type of tendinopathy [9]. However, intervention with NSAIDs may be related with gastrointestinal and cardio-vascular adverse effects [10]. Rather, load interventions, such as eccentric exercises (EE), are considered the first line treatment in AT rehabilitation [11]. EE have been shown to be effective in both reducing pain and improving functionality in patients with AT in several studies [12-14].

Cryotherapy is presented as an alternative intervention for patients with mid-portion AT and may decrease pain and normalise the blood flow [15]. Specifically, cryotherapy has demonstrated benefits alone or when combined with other therapies, such as compression in patients with AT [16].

Several studies have shown the efficacy of whole-body vibration training (WBVT) in endurance, triceps surae muscle strength, lower limb muscle flexibility, motor control and decreasing pain in subjects with AT $[17,18]$. In addition, Horstman et al. [18] carried out a 12-weeks WBVT program in 58 subjects with AT showing benefits in sonographic features, such as tendon structure and path, and pain intensity. Due to this, WBVT has been considered as an alternative intervention in AT patients.

Despite that Achilles EE programs are oriented to work eccentrically the lower limb, abdominal wall muscles are involved working in a coordinated manner for maintain the body stability and balance. Trunk muscles are partially comprised by transversus abdominis (TrA), external oblique (EO), internal oblique (IO) and rectus abdominis (RA), multifidus, pelvic floor muscles, erector spinae and quadratum lumborum, and they are morphologically different [19]. These muscles work synergistically transferring loads through the body, in both directions [20]. Regarding the deep trunk muscles, the TrA, multifidus, IO and pelvic floor muscles may be considered as deep stabilizers which support spine stability [21] and whose automatic activation provides a belt-like tension to the trunk which may be defined as "Core" [22]. Moreover, Hodges and Richardson [23] reported that TrA and oblique muscles work whenever there is a movement or an imbalance of the body, regardless of the direction of these forces. Considering the superficial trunk muscles, the RA, erector spinae, EO and quadratus lumborum may be considered as "global stabilizers" which support larger trunk movements using a quick and powerful torque [21]. Kim and Lee [24] reported that two lower limb muscle exercises, sit-up and leg raise showed an increase of the activation of the abdominal wall muscles, being the eccentric sit-up exercise the most efficient. Despite the eccentric activations of the lower limb muscles have been widely reported in patients with Achilles tendinopathy $[11,25,26]$, as well as the local effects of cryotherapy $[15,16]$ and vibration [18] on the Achilles tendon's symptoms and signs, there is a lack of knowledge regarding the global abdominal muscles activation during EE in conjunction with cryotherapy or vibration in patients with AT. In the abdominal complex area, rehabilitative ultrasound imaging (RUSI) has been widely used to evaluate abdominal wall muscles features, such as thickness, cross sectional area (CSA) and inter-rectus distance (IRD) [27-30]. Concretely, the RA thickness and IRD may be considered as key RUSI measurements of the global stabilizers morphology [31] and may be related to alterations in the lower limb neuromuscular control that result in abdominal wall adaptations [32].

The aim of the present study was to evaluate the RA muscle thickness and IRD in patients with chronic mid-portion AT who carried out an EE vibration program compared to an EE program with cryotherapy. It was hypothesized that an intervention with EE training in combination with vibration could be more beneficial for the abdominal muscle activation with respect to its combination with cryotherapy in patients with chronic mid-portion AT.

\section{Methods}

\section{Study Design}

The present study was a prospective, single-blinded, randomized, controlled clinical trial (registered at ClinicalTrials.gov as NCT03515148) evaluating individuals over a period of 12-weeks between January 2017 and January 2018, following the 
Consolidated Standards of Reporting Trials (CONSORT) guidelines.

\section{Participants}

In this study, 61 individuals diagnosed with mid-portion AT (age: $41.2 \pm 10$ years) were recruited and randomly divided in two groups (A and $\mathrm{B}$ ): group $A(n=30)$ received the EE program plus vibration and group $B(n=31)$ the EE program plus cryotherapy (Figure 1). The enrollment of subjects was performed by a specialized medical doctor with more than 10 years of experience in sport medicine. The selection criteria defined eligible subjects as those who: were aged 18-65 years, had had symptoms in the mid-portion of the Achilles tendon for at least 3 months [33], had a visual analog scale (VAS) pain score of at least 3 out of 10 points, had not received any physical therapy. Exclusion criteria were as follows: patients with any infection or systemic disease [12], a lower limb injury within the last 12 months, previous fracture [33] and negative experiences with one or more interventions in the past [34].

\section{Ethical statement}

The study was authorized by the ethics committee of Hospital Universitario de la Princesa, Madrid, Spain (approval code: 2828A). The study

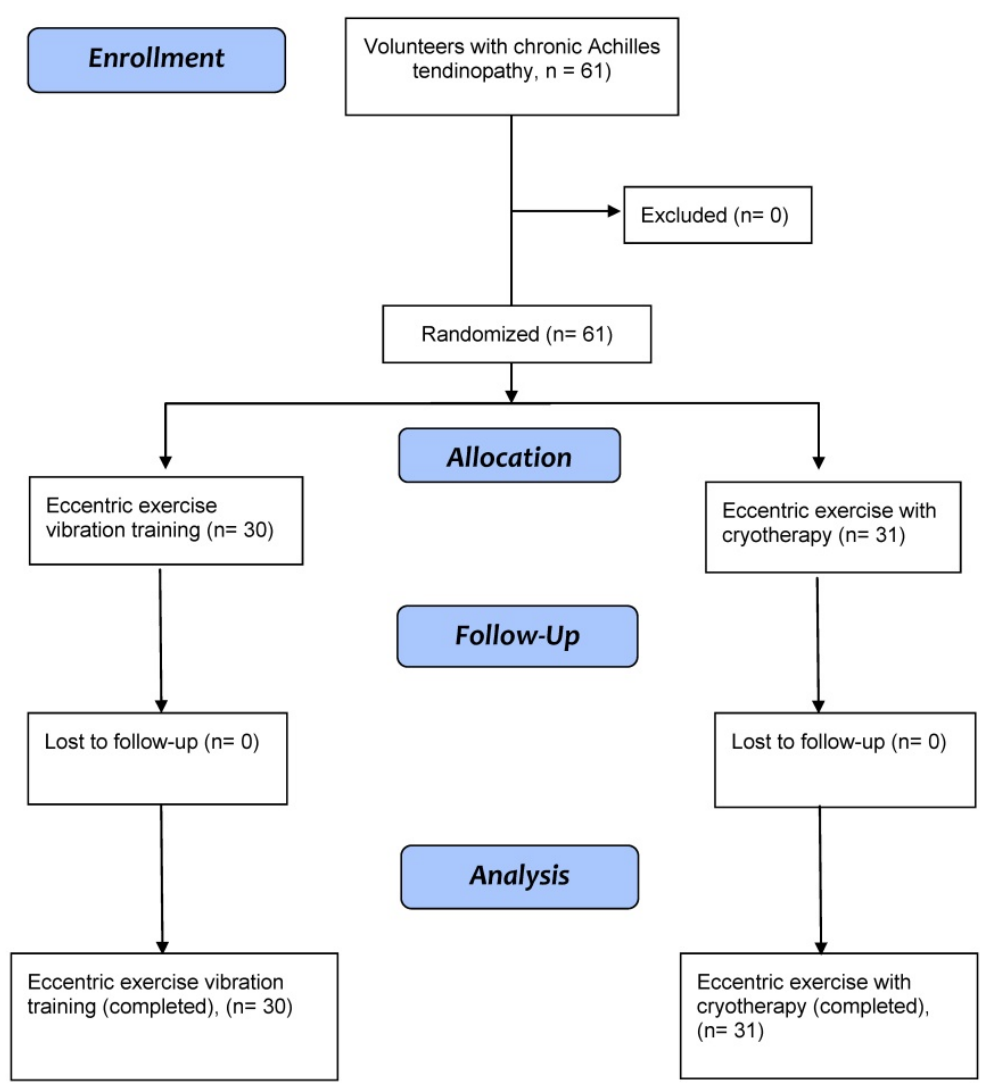

Figure 1. CONSORT flow algorithm outlining the participant enrollment, allocation, follow-up, and attrition numbers for this study. respects the Declaration of Helsinki for human experimentation [35]. All the participants in the study signed the informed consent form.

\section{Eccentric exercise intervention for both groups}

Both groups performed a 12-week EE program according to modified guidelines by Alfredson et al. [14]. The original protocol was composed of 90 repetitions, twice a day and 7 days per week. In the present study, all participants performed $90 \mathrm{EE}$ repetitions, by completing three sets of 15 repetitions in two training situations (45 repetitions with the knee fully extended and 45 repetitions with the knee slightly flexed). EE program was carried out in closed kinetic chain. The Template for Intervention Description and Replication (TIDieR) checklist was completed to ensure both interventions were reproducible and listed as per our registered protocol [36]. In addition, a protocol deviation was carried out due to week 4 follow-up was added even though not in the registered protocol. Participants' compliance to the protocol was self-reported in a written document.

\section{Eccentric exercise plus vibration program (A)}

Eccentric exercise plus vibration intervention was developed on a Power Plate My3 (Performance Health Systems, Northbrook, US) vibration platform following to the Alfredson et al. [14] EE protocol. The participants, who were included in this group, performed the EE program and were simultaneously placed in a standing position on the Power Plate system using a vibration frequency of $3 \mathrm{~Hz}$ and an amplitude of $4 \mathrm{~mm}$ for approximately 5 minutes (coinciding with the EE intervention duration) following the Hazell et al. [37] protocol.

\section{Eccentric exercise plus cryotherapy program (B)}

Before carrying out the EE program [14], the cryotherapy intervention was performed. Cryotherapy intervention was carried out by seating the patients and immersing their bare lower limb into a 70 -liter bucket containing $55 \mathrm{~cm}$-deep water at $8 \pm 2^{\circ} \mathrm{C}$ for 17 minutes [38]. After the immersion, the EE program was performed during 5 minutes following the previous described protocol [14].

\section{Outcome measures}

The ultrasound imaging assessment was carried out using a LogiQ P7 ultrasound system (GE Healthcare; UK) with a 4 to 13 
linear transducer (L6- 12- RS type; 38-mm footprint). Ultrasound assessments of the RA, ipsilateral to the $\mathrm{AT}$, and IRD were carried out in the supine position, with the transducer aligned with the umbilicus for the RA examination (Figure 2A) and just under the umbilicus for the IRD measurement (Figure 2B) in accordance with Whittaker et al. [27], who reported excellent interday and interexaminer reliability (intraclass correlation coefficients from 0.92 to 0.99 ) for these ultrasound measurements. Assessments were carried out prior to any intervention, and at 4 and at 12 weeks post-intervention, both with calf muscles at rest and in maximal isometric contraction. The mean of 3 repeated values was collected for each measurement with the transducer at the same point at the end of the expiration. Muscle thickness was described as the distance inside the caliper lines of each muscle (Figure 2A) and IRD was consider as the distance between the RA muscles (Figure 2B) [27]. ImageJ software (version 2.0) was used to analyze the images offline.

\section{Statistical analysis}

Statistical package for social sciences, version 23.0 software for Windows (IBM SPSS Statistics for Windows; NY: IBM Corp.) was employed for data analysis. An a error of 0.05 (95\% confidence interval) and a desired power of $80 \%$ ( $\beta$ error of 0.2 ) were used for all the statistical tests. The Shapiro-Wilk test was used for normality assumption. For the baseline comparison, the Student $\mathrm{t}$ test was used, considering the homogeneity of variance following Levene's test. A two-way analysis of variance (ANOVA) for repeated measures was employed to examine the intra-subject (pre and post) and inter-subject (treatment group) effects for the dependent variables.
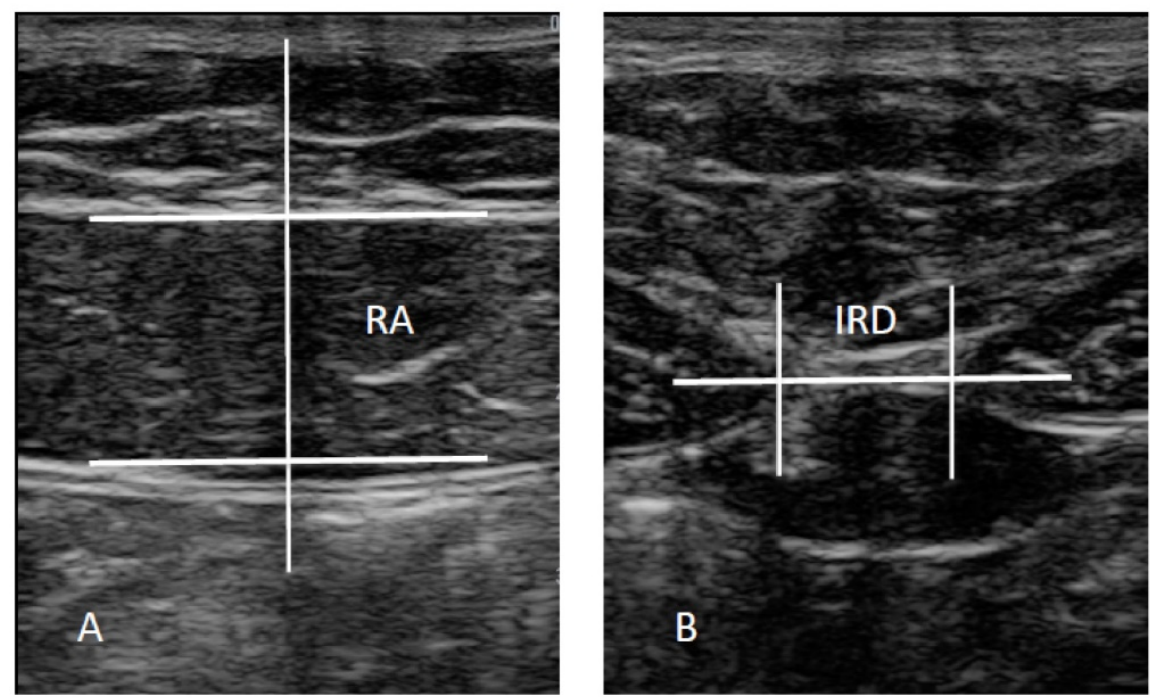

Figure 2. Ultrasound assessments. Abbreviations: IRD, inter-rectus distance; RA, rectus anterior.
Post-hoc analyses were carried out by means of Bonferroni's correction. The level of significance was set at $\mathrm{P}<0.05$.

\section{Results}

Sociodemographic data did not statistically differ between the two groups $(\mathrm{P}>0.05)$ (Table 1$)$ and all participants self-reported compliance to the protocol across the 12 weeks follow-up. IRD measures showed a significant $(\mathrm{P}<0.05)$ decrease at 4 and at 12-weeks in both groups (Table 2), but no significant differences $(\mathrm{P}>0.05)$ were observed between the intervention groups (Figure $3 \mathrm{~A}$ ). RA thickness was significantly increased $(\mathrm{P}<0.05)$ in measures at 4 and 12-weeks showed a significant increase in maximal isometric contraction and at rest in favor of the $\mathrm{EE}$ vibration program group (Figure 3B).

\section{Discussion}

To our knowledge, this is the first study that has evaluated RA thickness and IRD in patients with chronic mid-portion AT following an EE and vibration program compared to those following an EE program with cryotherapy.

\section{Inter-rectus distance}

Our findings showed an IRD decrease in both groups at 4 and 12-weeks. A IRD narrowing has been the target of contemporary rehabilitation interventions [31]. In addition, Lee and Hodges [39] reported that these treatment approaches are "based on the assumption that restore RA alignment restore the function" and get a better appearance [40]. Likewise, IRD is the goal of many women after the pregnancy, assuming that the abdominal wall may be weak at postpartum [41]. Several studies performed exercises focusing on the decrease of the IRD [31,42]. However, Lee and Hodges [39] suggest that a rehabilitation program should not be focused only in IRD treatment. Moreover, a IRD reduction could have counterproductive consequences for esthetic, alignment and function [39].

Based on our data, a lower limb EE program (with either vibration or cryotherapy) aimed at AT patients may also potentially benefit individuals with disturbances in abdominal wall muscles (e.g. low back pain, postpartum) as an alternative to conventional abdominal exercises, such abdominal crunches or planks. 


\section{Rectus abdominis thickness}

Lower limb EE have previously been shown to increase the activation of the abdominal wall muscles in healthy adults [24] and WBVT has also been shown to improve the motor units in muscles and extension strength of lower extremities [18], as well as muscle flexibility [43]. This is in agreement with our data, which show that RA thickness increases in both groups in favor of the EE vibration program. An increase in the thickness of the RA muscle, a fundamental structure between pelvis and upper limb, is beneficial in improving trunk stability, pressurizing the abdominal cavity and transferring loads from the lower limb to the upper limb.

\section{Current treatment of chronic mid-portion Achilles Tendinopathy}

Despite NSAIDs may help marginally decreasing tendinopathy symptoms, these pharmacologic interventions do not significant benefit patients with AT at long term [9], and may produce gastrointestinal or cardio-vascular adverse effects [10]. Exercise interventions have the advantage enhancing the Achilles tendon clinical symptoms [11,12]. A progressive muscle/tendon loading approach does seem to be beneficial, although it is unclear which programs lead to better clinical outcomes [13], notably because factors such as age, pain and tendinopathy site all influence load management [44]. Cook et al. [45] reported that manual therapies, electrotherapy and taping techniques should not substitute exercise programs, but complement them.

\section{IRD at Rest}

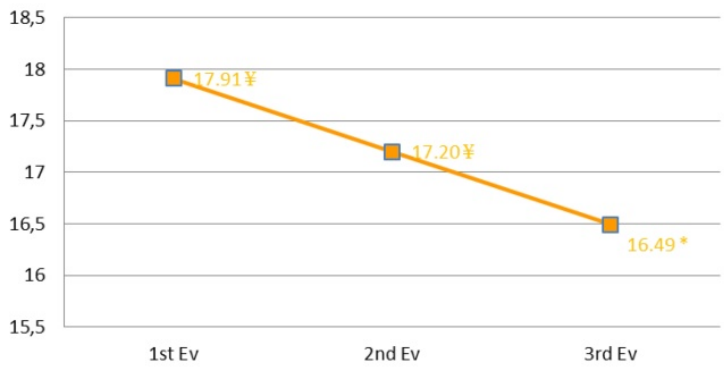

IRD at Contraction

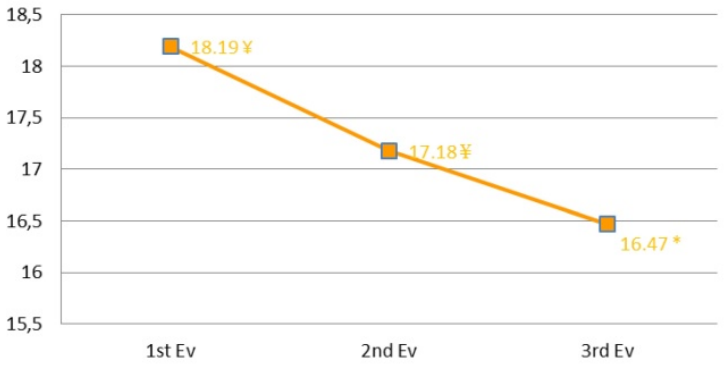

Table 1. Sociodemographic data

\begin{tabular}{llll}
\hline Data & Cryotherapy & EE Vibration Training & P value \\
\hline Sex & & & 0.645 \\
Men, (n, \%) & $5 \pm 16.13$ & $4 \pm 13.33$ & \\
Women, (n, \%) & $26 \pm 83.87$ & $26 \pm 86.66$ & \\
Age, y & $42.1 \pm 9.2$ & $41.1 \pm 8.2$ & 0.645 \\
Height & $1.72 \pm 0.0$ & $1.75 \pm 0.0$ & 0.698 \\
Weight & $73.63 \pm 9.38$ & $76.55 \pm 10.56$ & 0.285 \\
BMI, kg/m² & $24.8 \pm 2.4$ & $25.2 \pm 2.5$ & 0.442 \\
Injury time, mean (SD) & $4.4 \pm 2.6$ & $4.1 \pm 4.4$ & 0.145 \\
\hline
\end{tabular}

Abbreviations: body mass index, BMI

Table 2. Inter-recti distance and rectus abdominis thickness

\begin{tabular}{|c|c|c|c|c|}
\hline Measure (mm) & $\begin{array}{l}\text { Cryotherapy } \\
n=31\end{array}$ & $\begin{array}{l}\text { Eccentric Exercise } \\
\text { Vibration } \\
\text { Training } n=30\end{array}$ & $\begin{array}{l}\text { Intragroup } \\
\text { p-value }\end{array}$ & $\begin{array}{l}\text { intergrou } \\
\text { p p-value }\end{array}$ \\
\hline \multicolumn{3}{|l|}{ IRD Rest } & $.001^{*}$ & .068 \\
\hline Baseline & $18.41 \pm 6.7$ & $17.40 \pm 6.4$ & & \\
\hline 4 weeks & $18.04 \pm 6.6$ & $16.37 \pm 5.8$ & & \\
\hline 12weeks & $17.38 \pm 7.0$ & $15.61 \pm 5.9$ & & \\
\hline \multicolumn{3}{|c|}{ IRD Contraction } & $.001^{*}$ & .38 \\
\hline Baseline & $18.68 \pm 6.9$ & $17.70 \pm 7.3$ & & \\
\hline 4 weeks & $17.79 \pm 6.8$ & $16.57 \pm 6.5$ & & \\
\hline 12 weeks & $17.32 \pm 7.5$ & $15.62 \pm 5.7$ & & \\
\hline \multicolumn{3}{|c|}{ RA Thickness Rest } & $.001^{*}$ & $.002^{*}$ \\
\hline Baseline & $10.09 \pm 2.6$ & $10.30 \pm 2.2$ & & \\
\hline 4 weeks & $10.61 \pm 2.2$ & $10.56 \pm 2.0$ & & \\
\hline 12 weeks & $10.85 \pm 2.1$ & $11.83 \pm 2.1$ & & \\
\hline \multicolumn{3}{|c|}{ RA Thickness Contraction } & $.001^{*}$ & $.007^{*}$ \\
\hline Baseline & $10.06 \pm 2.8$ & $10.19 \pm 2.1$ & & \\
\hline 4 weeks & $10.58 \pm 2.4$ & $10.53 \pm 2.3$ & & \\
\hline 12weeks & $10.91 \pm 2.1$ & $11.91 \pm 2.0$ & & \\
\hline
\end{tabular}

Values are mean \pm SD unless otherwise indicated. Abbreviations: inter-recti distance, IRD; rectus anterior, RA
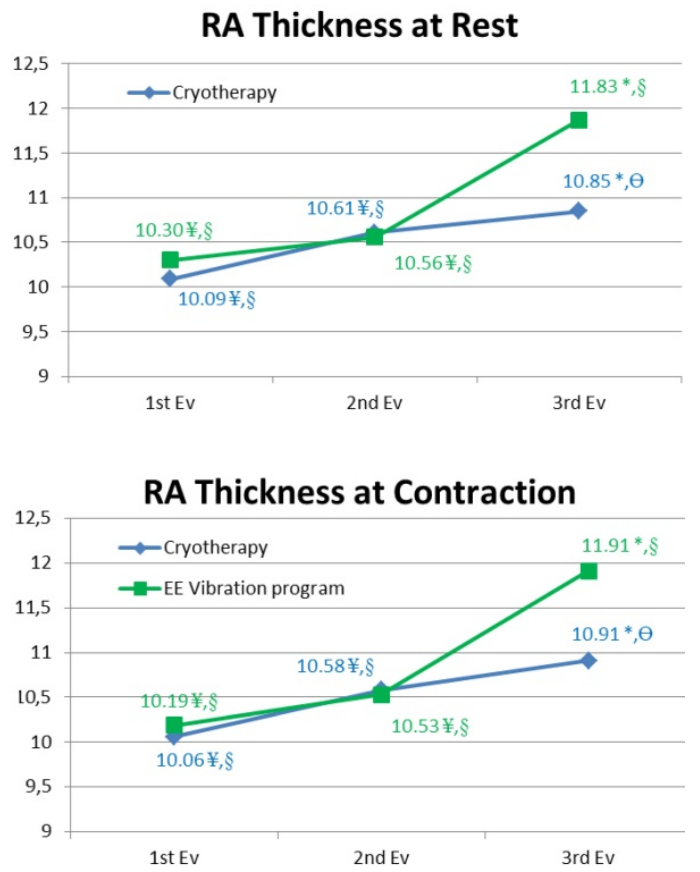

Figure 3. Inter-recti distance and rectus abdominis thickness. Abbreviations: IRD, inter-rectus distance; RA, rectus anterior. 


\section{Clinical considerations}

We indicate that a lower limb EE with WBVT program could be beneficial in patients with pathologies related to the abdominal wall muscles (e.g. low back pain, lumbopelvic pain and postpartum women), alongside more traditional approaches, such as the TrA approach.

\section{Limitations and futures studies}

The present study did not contain a suitable control group and the main finding can only be extrapolated to individuals with chronic Achilles mid-portion tendinopathy. Despite the accurate description of the EE protocol, the time under tension for the exercise and each repetition was not recorded. Sonoelastography, electromyography and other ultrasound modalities were not used and could be useful to provide more information about the muscle and the different tissues [46]. Despite the ultrasound measurements were performed according to Whittaker et al. [27] showing excellent interday and interexaminer reliability, a specific reliability assessment was not carried out. Futures studies could include postpartum women, sport populations and individuals with more relevant pathology, such low back pain and lumbopelvic pain. Finally, the clinical improvement of Achilles tendinopathy was not included in the present study and should be considered in future studies.

\section{Conclusions}

The present study showed a RA thickness increase in both groups in favor of the EE vibration program with respect to cryotherapy added to EE in short and mid term in maximal isometric contraction and at rest in subjects with chronic mid-portion AT. IRD was decreased in both groups without between-groups differences.

\section{Competing Interests}

The authors have declared that no competing interest exists.

\section{References}

1. Lopes AD, Hespanhol Junior LC, Yeung SS, Costa LOP. What are the main running-related musculoskeletal injuries? A Systematic Review. Sports Med. New Zealand; 2012 Oct:42(10):891-905.

2. Jarvinen TAH, Kannus P, Maffulli N, Khan KM. Achilles tendon disorders: etiology and epidemiology. Foot Ankle Clin. United States; 2005 Jun;10(2):255-66.

3. Albers IS, Zwerver J, Diercks RL, Dekker JH, Van den Akker-Scheek I. Incidence and prevalence of lower extremity tendinopathy in a Dutch general practice population: a cross sectional study. BMC Musculoskelet Disord. England; 2016 Jan;17:16.

4. Silbernagel KG, Gustavsson A, Thomee R, Karlsson J. Evaluation of lower leg function in patients with Achilles tendinopathy. Knee Surg Sports Traumatol Arthrosc. Germany; 2006 Nov;14(11):1207-17.

5. Munteanu SE, Barton CJ. Lower limb biomechanics during running in individuals with achilles tendinopathy: a systematic review. J Foot Ankle Res. England; 2011 May;4:15.
6. Magnan B, Bondi M, Pierantoni S, Samaila E. The pathogenesis of Achilles tendinopathy: a systematic review. Foot Ankle Surg. France; 2014 Sep;20(3):154-9.

7. Dean BJF, Gettings P, Dakin SG, Carr AJ. Are inflammatory cells increased in painful human tendinopathy? A systematic review. Br J Sports Med. England; 2016 Feb;50(4):216-20.

8. Dakin SG, Newton J, Martinez FO, Hedley R, Gwilym S, Jones N, et al. Chronic inflammation is a feature of Achilles tendinopathy and rupture. Br J Sports Med. 2017 Nov.

9. Skjong CC, Meininger AK, Ho SSW. Tendinopathy treatment: where is the evidence? Clin Sports Med. United States; 2012 Apr;31(2):329-50.

10. Roubille C, Martel-Pelletier J, Davy J-M, Haraoui B, Pelletier J-P. Cardiovascular adverse effects of anti-inflammatory drugs. Antiinflamm Antiallergy Agents Med Chem. United Arab Emirates; 2013;12(1):55-67.

11. Murphy M, Travers M, Gibson W, Chivers P, Debenham J, Docking S, et al. Rate of Improvement of Pain and Function in Mid-Portion Achilles Tendinopathy with Loading Protocols: A Systematic Review and Longitudinal Meta-Analysis. Sports Med. New Zealand; 2018 May.

12. Alfredson H, Pietila T, Jonsson P, Lorentzon R. Heavy-load eccentric calf muscle training for the treatment of chronic Achilles tendinosis. Am J Sports Med. United States; 1998;26(3):360-6.

13. Beyer R, Kongsgaard $M$, Hougs Kjaer B, Ohlenschlaeger $T$, Kjaer $M$, Magnusson SP. Heavy Slow Resistance Versus Eccentric Training as Treatment for Achilles Tendinopathy: A Randomized Controlled Trial. Am J Sports Med. United States; 2015 Jul;43(7):1704-11.

14. Stevens M, Tan C-W. Effectiveness of the Alfredson protocol compared with a lower repetition-volume protocol for midportion Achilles tendinopathy: a randomized controlled trial. J Orthop Sports Phys Ther. United States; 2014 Feb;44(2):59-67.

15. Haslerud S, Lopes-Martins RA, Frigo L, Bjordal JM, Marcos RL, Naterstad IF, Magnussen LH, Joensen J. Low-Level Laser Therapy and Cryotherapy as Mono- and Adjunctive Therapies for Achilles Tendinopathy in Rats. Photomed Laser Surg. 2017 Jan;35(1):32-42.

16. Knobloch K, Grasemann R, Spies M, Vogt PM. Midportion achilles tendon microcirculation after intermittent combined cryotherapy and compression compared with cryotherapy alone: a randomized trial. Am J Sports Med. United States; 2008 Nov;36(11):2128-38.

17. Hilgers C, Mundermann A, Riehle H, Dettmers C. Effects of whole-body vibration training on physical function in patients with multiple sclerosis. NeuroRehabilitation. Netherlands; 2013;32(3):655-63.

18. Horstmann T, Jud HM, Frohlich V, Mundermann A, Grau S. Whole-body vibration versus eccentric training or a wait-and-see approach for chronic Achilles tendinopathy: a randomized clinical trial. J Orthop Sports Phys Ther. United States; 2013 Nov;43(11):794-803.

19. Brown SHM, Ward SR, Cook MS, Lieber RL. Architectural analysis of human abdominal wall muscles: implications for mechanical function. Spine (Phila Pa 1976) [Internet]. 2011;36(5):355-62.

20. Richardson CA, Snijders CJ, Hides JA, Damen L, Pas MS, Storm J. The relation between the transversus abdominis muscles, sacroiliac joint mechanics, and low back pain. Spine (Phila Pa 1976). 2002 Feb;27(4):399-405.

21. Huxel Bliven KC, Anderson BE. Core Stability Training for Injury Prevention. Sport Heal A Multidiscip Approach. 2013 Nov;5(6):514-22.

22. Kibler W Ben, Press J, Sciascia A. The role of core stability in athletic function. Sports Med. 2006;36(3):189-98.

23. Hodges PW, Richardson CA. Contraction of the abdominal muscles associated with movement of the lower limb. Phys Ther. United States; 1997 Feb;77(2):132-4.

24. Kim K, Lee T. Comparison of muscular activities in the abdomen and lower limbs while performing sit-up and leg-raise. Journal of Physical Therapy Science. 2016;8: 491-4.

25. Yu J. Comparison of Lower Limb Muscle Activity during Eccentric and Concentric Exercises in Runners with Achilles Tendinopathy. J Phys Ther Sci [Internet]. 2014;26(9):1351-3.

26. Azevedo LB, Lambert MI, Vaughan CL, O'Connor CM, Schwellnus MP. Biomechanical variables associated with Achilles tendinopathy in runners. $\mathrm{Br} \mathrm{J}$ Sports Med [Internet]. 2009;43(4):288-92.

27. Whittaker JL, Warner MB, Stokes M. Comparison of the Sonographic Features of the Abdominal Wall Muscles and Connective Tissues in Individuals With and Without Lumbopelvic Pain. J Orthop Sport Phys Ther [Internet]. 2013;43(1):11-9.

28. Teyhen DS, Gill NW, Whittaker JL, Henry SM, Hides JA, Hodges P. Rehabilitative ultrasound imaging of the abdominal muscles. J Orthop Sports Phys Ther [Internet]. 2007;37(8):450-66.

29. Kiesel KB, Uhl T, Underwood FB, Nitz AJ. Rehabilitative ultrasound measurement of select trunk muscle activation during induced pain. Man Ther. Scotland; 2008;13(2):132-8.

30. Mota P, Pascoal AG, Sancho F, Carita AI, Bø K. Reliability of the inter-rectus distance measured by palpation. Comparison of palpation and ultrasound measurements. Man Ther [Internet]. 2013;18(4):294-8.

31. Pascoal AG, Dionisio S, Cordeiro F, Mota P. Inter-rectus distance in postpartum women can be reduced by isometric contraction of the abdominal muscles: a preliminary case-control study. Physiotherapy. England; 2014 Dec;100(4):344-8. 
32. Whittaker JL, Emery CA. Impact of the FIFA $11+$ on the structure of select muscles in adolescent female soccer players. Phys Ther Sport [Internet]. 2015;16(3):228-35.

33. Alfredson $\mathrm{H}$, Cook J. A treatment algorithm for managing Achilles tendinopathy: new treatment options. British Journal of Sports Medicine. 2007;41: 211-6.

34. Astrom M, Gentz CF, Nilsson P, Rausing A, Sjoberg S, Westlin N. Imaging in chronic achilles tendinopathy: a comparison of ultrasonography, magnetic resonance imaging and surgical findings in 27 histologically verified cases. Skeletal Radiol. Germany; 1996 Oct;25(7):615-20.

35. Holt GR. Declaration of Helsinki-the world's document of conscience and responsibility. South Med J [Internet]. 2014;107(7):407.

36. Hoffmann TC, Glasziou PP, Boutron I, Milne R, Perera R, Moher D, et al. Better reporting of interventions: template for intervention description and replication (TIDieR) checklist and guide. BMJ. 2014;348:g1687-g1687.

37. Hazell TJ, Jakobi JM, Kenno KA. The effects of whole-body vibration on upper- and lower-body EMG during static and dynamic contractions. Appl Physiol Nutr Metab = Physiol Appl Nutr Metab. Canada; 2007 Dec;32(6):1156-63.

38. khanmohammadi R, Someh M, Ghafarinejad F. The Effect of Cryotherapy on the Normal Ankle Joint Position Sense. Asian Journal of Sports Medicine. 2011;2: 91-8.

39. Lee D, Hodges PW. Behavior of the Linea Alba During a Curl-up Task in Diastasis Rectus Abdominis: An Observational Study. J Orthop Sports Phys Ther. United States; 2016 Jul;46(7):580-9.

40. Nahas FX. An aesthetic classification of the abdomen based on the myoaponeurotic layer. Plast Reconstr Surg. United States; 2001 Nov;108(6):1787.

41. Liaw L-J, Hsu M-J, Liao C-F, Liu M-F, Hsu A-T. The relationships between inter-recti distance measured by ultrasound imaging and abdominal muscle function in postpartum women: a 6-month follow-up study. J Orthop Sports Phys Ther. United States; 2011 Jun;41(6):435-43.

42. Mota P, Pascoal AG, Carita AI, Bo K. The Immediate Effects on Inter-rectus Distance of Abdominal Crunch and Drawing-in Exercises During Pregnancy and the Postpartum Period. J Orthop Sports Phys Ther. United States; 2015 Oct; $45(10): 781-8$

43. Kinser AM, Ramsey MW, O'Bryant HS, Ayres CA, Sands WA, Stone MH. Vibration and stretching effects on flexibility and explosive strength in young gymnasts. Med Sci Sports Exerc. United States; 2008 Jan;40(1):133-40.

44. Rompe JD, Nafe B, Furia JP, Maffulli N. Eccentric Loading, Shock-Wave Treatment, or a Wait- and-See Policy for Tendinopathy of the Main Body of Tendo Achillis: A Randomized Controlled Trial. Am J Sports Med. 2007;35(3):374-83

45. Cook JL, Stasinopoulos D, Brismee J-M. Insertional and mid-substance Achilles tendinopathies: eccentric training is not for everyone - updated evidence of non-surgical management. The Journal of manual \& manipulative therapy. England; 2018;2: 119-22.

46. Bunce SM, Hough AD, Moore AP Measurement of abdominal muscle thickness using M-mode ultrasound imaging during functional activities. Man Ther [Internet]. 2004;9(1):41-4. 